\title{
The Local Universe: Galaxies in 3D
}

\author{
Bärbel S. Koribalski \\ CSIRO Astronomy and Space Science, Australia Telescope National Facility, \\ P. O. Box 76, Epping, NSW 1710, Australia \\ email: Baerbel.Koribalski@csiro.au
}

\begin{abstract}
Here I present results from individual galaxy studies and galaxy surveys in the Local Universe with particular emphasis on the spatially resolved properties of neutral hydrogen gas. The 3D nature of the data allows detailed studies of the galaxy morphology and kinematics, their relation to local and global star formation as well as galaxy environments. I use new 3D visualisation tools to present multi-wavelength data, aided by tilted-ring models of the warped galaxy disks. Many of the algorithms and tools currently under development are essential for the exploration of upcoming large survey data, but are also highly beneficial for the analysis of current galaxy surveys.
\end{abstract}

Keywords. galaxies: individual (M 83), evolution \& formation, radio surveys - technology: interferometry, wide-field phased array feeds

\section{Introduction}

Analysing the well-resolved stellar and gas kinematics of disk galaxies provides insights into their rotational and non-rotational components, both of which can be measured as a function of radius and disk height. The resulting rotation curves reflect the overall mass distribution of galaxies (eg., Bosma 2004), including their very large dark matter halos, which extend well beyond the observed disks (e.g., Jones et al. 1999; Warren et al. 2004; Kreckel et al. 2011; Koribalski \& Lopez-Sanchez 2009; Westmeier et al. 2011, 2013). High-resolution spectroscopic data cubes (targeting, eg., the $\mathrm{H} \mathrm{I}, \mathrm{CO}$, and $\mathrm{H} \alpha$ spectral lines) also allow us to determine the 3D shape of galaxies, including their warped disks. The various shapes of 3D models derived with TiRiFiC, the Tilted Ring Fitting Code, are nicely illustrated by Józsa (2007). I then construct 3D visualisations of the observed galaxies based on multi-wavelength imaging and spectral line cubes at various angular resolutions, allowing me to analyse and improve the 3D representation of each galaxy until it reflects the observed data and knowledge derived from the data. Another benefit of the re-constructed 3D particle model is the ability to view each galaxy from any angle and produce fly-through movies (as presented for the galaxy M 83). In this first attempt, I used high-resolution optical and ultraviolet images as well as $\mathrm{H}$ I spectral line cubes from the Australia Telescope Compact Array (ATCA).

Optical galaxy surveys with ever more powerful integral fields units (IFUs), such as ATLAS-3D (Cappellari et al. 2011), CALIFA (Sánchez et al. 2012) and SAMI (Allen et al. 2014), are now delivering high-resolution data cubes. Radio synthesis telescopes like the ATCA, which consists of six 22-m dishes, have been recording large spectral line data cubes for over two decades. The ATCA primary beam is $\sim 0.5$ degr at $21-\mathrm{cm}$; H I emission from nearby galaxies within this large field of view can be mapped with angular resolutions of up to $\sim 10$ arcsec and velocity resolution of less than $1 \mathrm{~km} \mathrm{~s}^{-1}$. Much higher resolutions are typically used when observing molecular lines (ATCA receivers cover frequencies from 1 to $105 \mathrm{GHz}$ observable with two $2 \mathrm{GHz}$-wide bands; Wilson et al. 2011). 


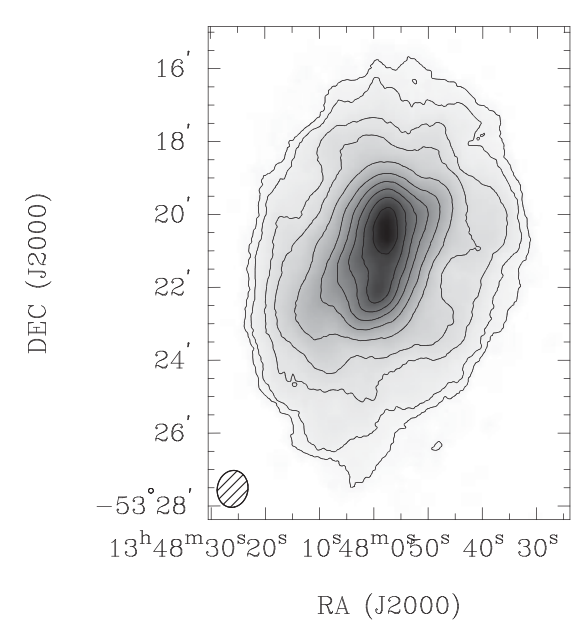

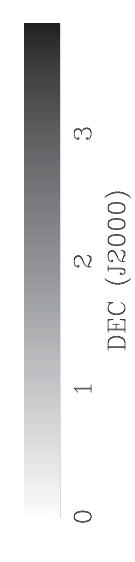

RA (J2000)

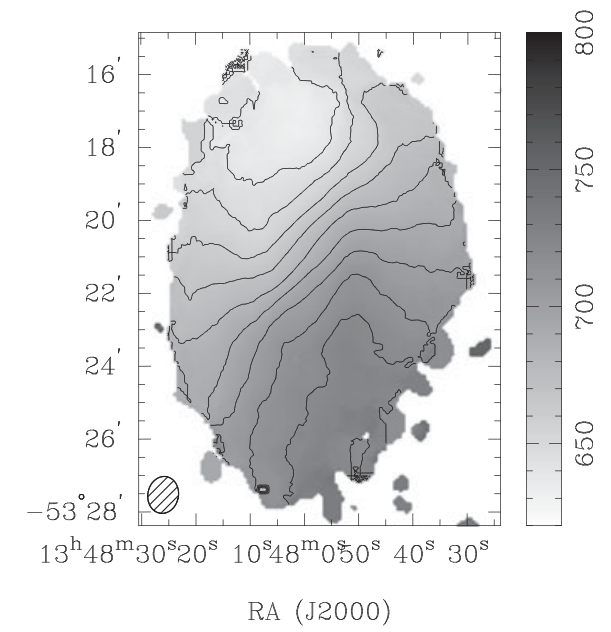

Figure 1. ATCA H I moment maps of the low-surface brightness galaxy ESO174-G?001 obtained as part of the Local Volume HI Survey (LVHIS) project (Koribalski 2010). The H I distribution (left) extends far beyond the stellar disk, while the mean H I velocity field (right) shows a regular, but rather twisted rotation pattern.

ASKAP, the Australian Square Kilometre Array Pathfinder (Johnston et al. 2008), which will be fully equipped with novel wide-field phased array feeds (PAFs), is just starting to deliver the first well-resolved H I maps of nearby galaxies and groups. We are currently observing with six of the 36 antennas, forming nine beams (each with FWHM $\sim 1$ degr) which can be placed anywhere within the 30 sq degr field-of-view. After successful ASKAP H I mapping the the Sculptor Group galaxies NGC 253 and NGC 247 plus the area in between this wide pair, we are now imaging the nearby gas-rich galaxy group IC 1459 and its environment (Serra et al. 2015). The recording bandwidth is $300 \mathrm{MHz}$ divided into around 17000 channels giving a velocity resolution of $4 \mathrm{~km} \mathrm{~s}^{-1}$. The angular resolution of the current 6-antenna array, known as the Boolardy Engineering Test Array (BETA; Hotan et al. 2014) is $~ 1$ arcmin; the frequency coverage is 700 to $1800 \mathrm{MHz}$.

ASKAP is a powerful 21-cm survey machine and will - once fully equipped with the equivalent of wide-field IFUs - be mapping the neutral hydrogen line in emission and absorption over the whole southern sky and a good fraction of the northern sky. Several large ASKAP H I surveys are planned. Here I briefly introduce WALLABY, a 21-cm survey of the sky $\left(\delta<+30^{\circ} ; z<0.26\right)$ which in about one year of observing time will detect more than 500000 galaxies in the H I spectral line (Duffy et al. 2012, Koribalski 2012 b), ie. a factor $\sim 20$ more than currently catalogued. Novel Chequerboard PAFs are providing $30 \mathrm{sq}$ degr instantaneous field-of-view. In WALLABY $\sim 1000$ galaxies will have $\mathrm{H}$ I diameters larger than $5 \operatorname{arcmin}(>10$ beams) and $\sim 5000$ galaxies will have majoraxis $\mathrm{H}$ I diameters greater than 2.5 arcmin ( $>5$ beams), allowing us to study in detail their morphology, kinematics and mass distribution. The number would rise to $1.6 \times 10^{5}$ galaxies if all 36 ASKAP antennas could be used; the additional six antennas provide baselines up to $6 \mathrm{~km}$, resulting in an angular resolution of $10^{\prime \prime}$. Creating highly reliable and complete source catalogs requires sophisticated source-finding algorithms as well as accurate source parametrisation. We are aiming to achieve this with our new Source Finding Application (SoFiA; Serra et al. 2014) and H I profile fitting with the versatile "Busy Function" (Westmeier et al. 2014). For an overview on continuum and spectral line source finding see the PASA Special Issue (Koribalski 2012a). 


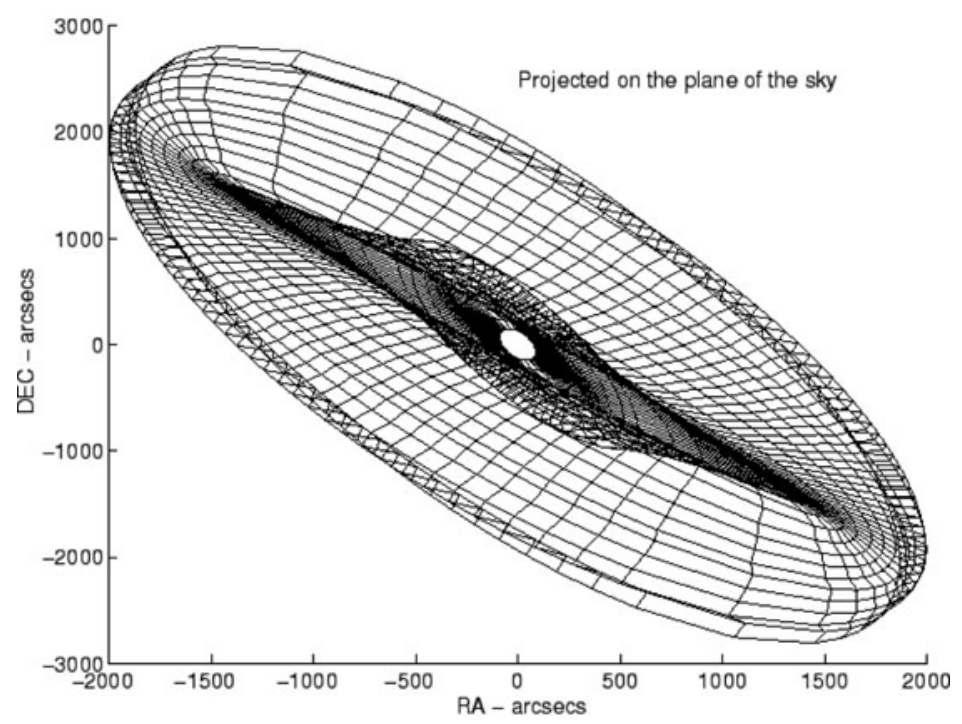

Figure 2. Example 3D model of a warped spiral galaxy.

\section{Kinematic Modelling}

A common way of analysing a galaxy's H I velocity field is to carefully fit tilted ring models (de Blok et al. 2008, Oh et al. 2010). The resulting residual velocity field is a good indicator of the goodness of fit and any remaining peculiar (non-rotational) structures. Tilted ring fits retrieve structural parameters, such as position angle, inclination and rotational velocity, as a function of radius when a galaxy is moderately well resolved. Several well-tested algorithms/programs exist, most of which are applied to the $2 \mathrm{D}$ velocity field. In some cases, for example edge-on disks and large well-resolved $\mathrm{H}$ I disks of galaxies, 3D modelling of the $\mathrm{H}$ I data cubes is of advantage (eg., Kamphuis et al. 2013).

The WALLABY kinematics working group (led by Kristine Spekkens) has started to create a modular package that allows to input spectral data cubes of individual galaxies, calculate $\mathrm{H}$ I velocity fields (eg., as shown in Fig. 1) and fit them with one or more of the algorithms described below. We base this approach on our successful SoFiA package (Serra et al. 2014). Assuming circular symmetry, the fitted rings allow an accurate description of warped galaxy disks where both inclination, $i(r)$, and position angle, $P A(r)$, vary with radius $r$. This is necessary to correctly deproject the measured rotational velocities to derive the galaxy rotation curve, $v_{\text {rot }}^{i}(r)$. Rogstad et al. (1974) introduced the tilted-ring model fitting, which is widely used through the Rotcur program and applied to $2 \mathrm{D}$ velocity fields. TiRiFiC uses spectroscopic data cubes to obtain tilted ring model fits of galaxies; it is now available as a stand-alone routine. This approach is essential for galaxy disks seen edge-on and allows sophisticated modelling of the disk thickness and surface brightness distribution (Kamphuis et al. 2013, 2014). DiskFit was created for the kinematic modeling of barred spiral galaxies. It does not allow the fitting of warped disk where the inclination and position angles vary with radius. DiskFit buids on Velfit (Spekkens \& Sellwood 2007). The Kinemetry technique (Krajnovic et al. 2006) was devised to model the stellar velocity fields of early-type galaxies. 


\section{The Spiral Galaxy M 83}

In the following I will use the grand-design spiral galaxy as an example of $3 \mathrm{D} \mathrm{H} \mathrm{I}$ mapping. The $\mathrm{H}$ I envelope of M 83 is much more extended than the well-known stellar disk. A prominent tidal arms hints at gravitational interactions with its dwarf galaxy neighbours while the western edge of the gas appears compressed, possibly affected by ram pressure stripping. The $\mathrm{H}$ I velocity field shows both the rotation and the changing orientation of the gaseous disk. By modelling the disk we are able to reconstruct the true $3 \mathrm{D}$ shape of a galaxy and place it within the group volume. In Section 5 I give more details on the $3 \mathrm{D}$ visualisation of stars and gas using the ray-tracing software Splotch. Currently, we can model several hundred galaxies in this way. Future large-scale ASKAP $\mathrm{H}$ I surveys will allow us to do this for several thousand galaxies, obtaining a true $3 \mathrm{D}$ dynamic picture of the nearby Universe.

Our large-scale H I mosaic of the beautiful, grand design spiral galaxy M 83 (NGC 5236, HIPASS J1337-29), obtained by combining ATCA interferometric and Parkes single-dish data (see Fig. 3), reveals an enormous H I disk with a diameter of $\sim 80 \mathrm{kpc}$, several times larger than M 83's optical Holmberg diameter. Here we adopt a distance of $D=4.5 \mathrm{Mpc}$. While the inner disk of M 83 rotates remarkably regular, the H I gas dynamics appear increasingly peculiar towards the outer regions which show clear signs of tidal disruption. The most prominent tidal features of M 83 are the one-sided outer H I arm which can be traced over 180 degr from the western to the eastern side, and the spectacular stellar stream, consisting of mainly old stars, to the north; their origin and possible relation are explored. M 83 is surrounded by numerous dwarf galaxies and, given its dynamical mass of about $5 \times 10^{11} \mathrm{M}_{\odot}$, is likely to attract and accrete them in regular intervals.

From a very deep optical image of M 83 by Malin \& Hadley (1997) we estimate a stellar diameter of $\sim 22^{\prime} \times 19^{\prime}$ (ie, $29 \mathrm{kpc} \times 25 \mathrm{kpc}$ ) for the disk at a $B$-band surface brightness of $\sim 28 \mathrm{mag} \operatorname{arcsec}^{-2}$. For comparison, the optical $B_{25}$ diameter of $\mathrm{M} 83$ is $12.9^{\prime} \times 11.5^{\prime}$ (de Vaucouleurs et al. 1991). Beyond the faint stellar disk, the deep optical image reveals three peculiar features: a prominent north-western stellar stream

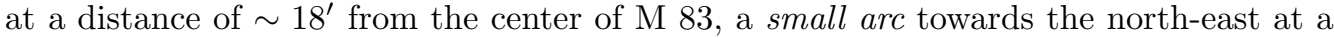
distance of $\sim 11^{\prime}$, and a southern ridge. GALEX FUV emission is clearly detected in the southern ridge as well as the western part of the small arc which we identified in the deep optical and $\mathrm{H}$ I images of M 83. (see Fig. 3). The mean $B$-band surface brightness of the extended stellar stream to the north of $\mathrm{M} 83$ is around $27 \mathrm{mag} \operatorname{arcsec}^{-2}$. It covers an area of about $10 \operatorname{arcmin}^{2}$ or about $17 \mathrm{kpc}^{2}$. The $\mathrm{H}$ I distribution (0. moment) of M 83 is most remarkable. No longer does this grand-design spiral look regular and undisturbed. The H I maps show streamers, irregular enhancements, an asymmetric tidal arm, diffuse emission, and a highly twisted velocity field, much in contrast to its regular appearance in short-exposure optical images. M 83's $\mathrm{H}$ I mass $\left(\mathrm{M}_{\mathrm{HI}}=7.8( \pm 0.5) \times 10^{9} \mathrm{M}_{\odot}\right)$ is more than twice that of an $\mathrm{M}_{\mathrm{HI}}^{*}$ galaxy. The $\mathrm{H}$ I appearance of $\mathrm{M} 83$ clearly suggests that it has been and possibly still is interacting with neighbouring dwarf galaxies. The effect of this interaction on the dwarfs can of course be rather devastating; it is quite likely that M 83 has accreted dwarf galaxies in the past as the stellar and gaseous streams/tails.

The eastern-most $\mathrm{H}$ I emission of M 83 which forms part of its peculiar, outer arm lies $\sim 34.5^{\prime}$ or $45 \mathrm{kpc}$ away from the center of $\mathrm{M} 83$. And the the dwarf irregular galaxy NGC 5264 lies at a projected distance of only $25.5^{\prime}$ or $33 \mathrm{kpc}$ from the eastern $\mathrm{H}$ I edge of $\mathrm{M}$ 83. For NGC 5264 we measure $\mathrm{M}_{\mathrm{HI}}=6 \times 10^{7} \mathrm{M}_{\odot}$ and calculate a total dynamical mass of $\sim 1.2 \times 10^{9} \mathrm{M}_{\odot}$. We also detected the dwarf irregular galaxy UGCA 365 to the north of $\mathrm{M} 83$, just outside its large $\mathrm{H}$ I envelope. We measure $\mathrm{M}_{\mathrm{HI}}=1.5 \times 10^{7} \mathrm{M}_{\odot}$ and a total dynamical mass of $\sim 1.2 \times 10^{9} \mathrm{M}_{\odot}$. 


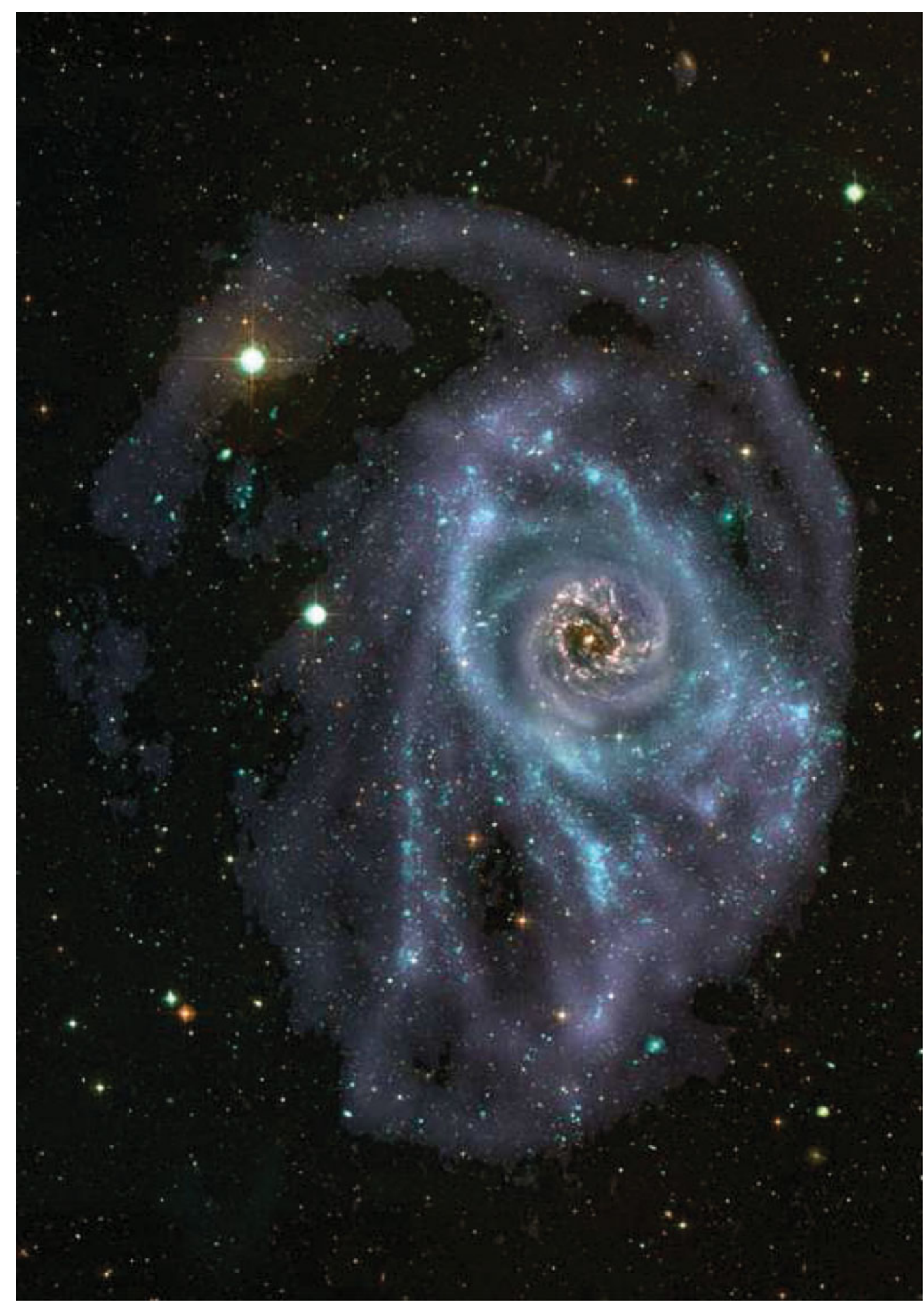

Figure 3. Multi-wavelength image of the spiral galaxy M 83. The large neutral hydrogen (H I) disk, shown in blue, was mapped with the ATCA and extends well beyond the stellar disk. For the color composition we also used GALEX NUV+FUV (light blue), DSS $R$-band (green), and 2MASS $J$-band (red) images. (Image credit: Ángel R. López-Sánchez). 


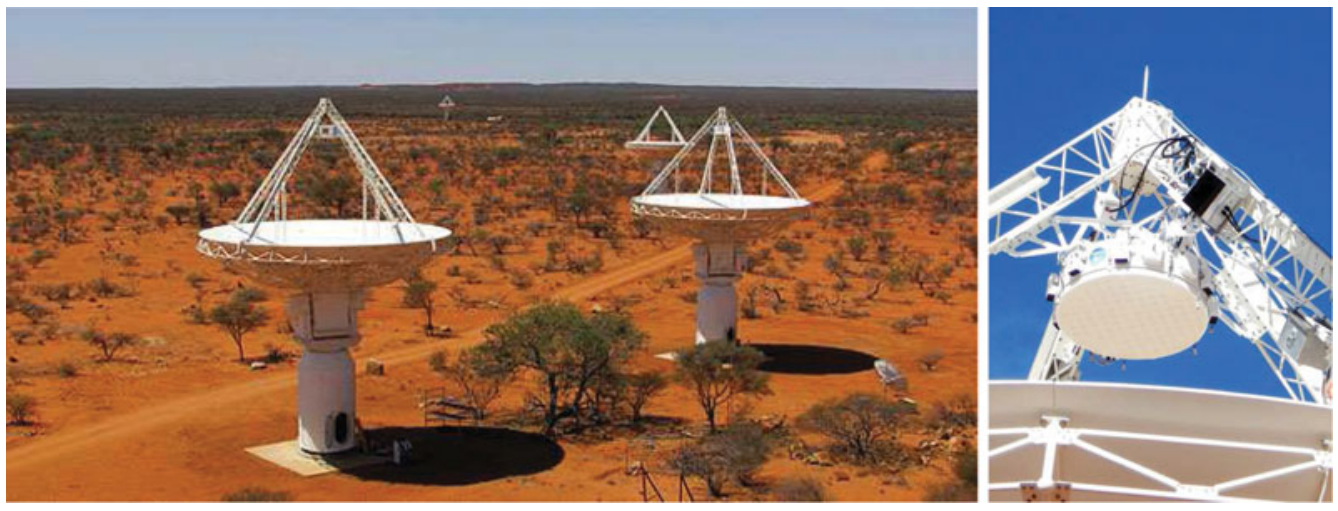

Figure 4. - (Left) Three of the 36 ASKAP antennas at the Murchison Radio astronomy observatory (MRO) in Western Australia. - (Right) The first Mk II PAF was installed on ASKAP antenna 29 in September 2014. (Image credits: Ant Schinckel, CSIRO).

For comparison, the $\mathrm{H}$ I disk of the Circinus galaxy has a diameter of $\sim 80^{\prime}$ or $\sim 100$ kpc at an adopted distance of $4.2 \mathrm{Mpc}$ (For, Koribalski \& Jarrett 2012), 5× larger than the extrapolated Holmberg diameter of $\sim 17^{\prime}$ (Freeman et al. 1977).

\section{SKA Pathfinder HI Surveys}

We have come a long way since the discovery of the 21-cm spectral line by Ewen \& Purcell in 1951. Nevertheless, detecting H I emission in a Milky Way-like galaxy at redshift $z=1$ will require the Square Kilometre Array (SKA; Rawlings ?; Obreschkow et al. 2011). Several SKA pathfinder and precursor telescopes are currently testing new technologies and used to develop reliable data processing pipelines. Much of the data is too large to store long-term, so data processing, quality control, imaging and source finding has to be done as the raw data is being recorded.

Several tens of thousands galaxies have so far been detected in the 21-cm H I line, the vast majority with single dish radio telescopes (e.g., Meyer et al. 2004, Springob et al. 2005, Wong et al. 2006, Haynes et al. 2011). The intrinsic faintness of the electron spinflip transition of neutral atomic hydrogen (rest frequency $1.42 \mathrm{GHz}$ ) makes it difficult to detect $\mathrm{H}$ I emission from individual galaxies at large distances. To study the $\mathrm{H}$ I content of galaxies and diffuse $\mathrm{H}$ I filaments between galaxies, we need radio synthesis telescopes with large collecting areas, low-noise receivers and large fields of view.

ASKAP consists of $36 \times 12-\mathrm{m}$ antennas and is located in the Murchison Shire of Western Australia (see Fig. 4). Of the 36 antennas, 30 are located within a circle of $\sim 2 \mathrm{~km}$ diameter, while six antennas are at larger distances providing baselines up to $6 \mathrm{~km}$. Six ASKAP dishes are currently equipped with first-generation (Mk I) Chequerboard PAFs. The instantaneous field-of-view of the ASKAP PAFs is $5.5 \mathrm{deg} \times 5.5 \mathrm{deg}$, ie. 30 square degrees, making ASKAP a 21-cm survey machine. The WSRT APERTIF upgrade employs Vivaldi PAFs, delivering a field-of-view of 8 square degrees (Verheijen et al. 2008).

WALLABY, the Widefield ASKAP L-band Legacy All-sky Blind surve $Y$ (led by me and Lister Staveley-Smith; see Koribalski et al. 2009), will cover $75 \%$ of the sky $\left(-90^{\circ}<\right.$ $\delta<+30^{\circ}$ ) over a frequency range from 1.13 to $1.43 \mathrm{GHz}$ (corresponding to $-2000<c z<$ $77,000 \mathrm{~km} \mathrm{~s}^{-1}$ ) at resolutions of $30^{\prime \prime}$ and $4 \mathrm{~km} \mathrm{~s}^{-1}$. WALLABY will be carried out using the inner 30 antennas of ASKAP, which provide excellent $u v$-coverage and baselines up to $2 \mathrm{~km}$. High-resolution (10") ASKAP H I observations using the full 36-antenna array will 
require further computing upgrades. WNSHS, the Westerbork Northern Sky HI Survey (led by Guyla Józsa), will cover a large fraction of the northern sky $\left(\delta>+27^{\circ}\right)$ with APERTIF over the same frequency range as WALLABY with ASKAP. Both H I surveys combined will achieve a true all-sky survey with unprecedented resolution and depth. The science goals of both surveys are well developed and complement, as well as enhance, each other. For a summary see Koribalski (2012b). WALLABY and WNSHS are made possible by the development of phased array feeds, delivering a much larger field-ofview than single feed horns or multi-beam systems. WALLABY will take approximately one year (ie 8 hours per pointing) and deliver an rms noise of $1.6 \mathrm{mJy}$ beam $^{-1}$ per $4 \mathrm{~km} \mathrm{~s}^{-1}$ channel. WALLABY is a precursor for future $\mathrm{H}$ I surveys with SKA Phase I and II, exploring the role of atomic hydrogen in galaxy formation and evolution. Using the $\mathrm{H}$ I-detected galaxies over two thirds of sky we will be able to unveil their largescale structures and cosmological parameters. For nearby galaxies, we can detect their extended, low-surface brightness disks as well as gas streams and filaments between galaxies.

\section{3D Visualisation of gas and stars in galaxies}

Animations of several thousand HIPASS galaxies show their $3 \mathrm{D}$ distribution in the nearby Universe $(z<0.03)$, based on their measured positions, velocities / distances and H I masses (created by Mark Calabretta and available on-line). Large-scale structures such as the Supergalactic Plane and the Local Void (see Koribalski et al. 2004) are clearly visible. The resolution of HIPASS, the H I Parkes All Sky Survey, is 15.5 arcmin and $18 \mathrm{~km} \mathrm{~s}^{-1}$. Once WALLABY data are in hand (resolution $30 \operatorname{arcsec}$ and $4 \mathrm{~km} \mathrm{~s}^{-1}$ ), we should be able to create animations of 500000 galaxies as well as detailed 3D models of $~ 5000$ galaxies (ie, all HIPASS galaxies) by a 3D multi-wavelength rendering of the respective galaxy. Multi-wavelength images and spectral line data cubes of galaxies allow us to measure their stellar, gas and dark matter properties. Visualisation packages such as KARMA provide a range of tools to interactively view $2 \mathrm{D}$ and $3 \mathrm{D}$ data sets as well as apply mathematical operations. This allows not only the quick inspection and evaluation of multiple images, spectra and cubes, but also the production of beautiful multi-color images and animations. To improve our understanding of galaxy formation and evolution, we need to include models and theoretical knowledge together with observations of galaxy disks and halos. By fitting and modelling the observed gas distribution and kinematics of extended galaxy disks, we derive their 3D shapes and rotational velocities. Visualisation can then be employed to combine the actual data with our derived knowledge to re-construct the most likely 3D representation of each galaxy. By adding time as the fourth dimension one can also visualize the evolution of galaxies and the Universe (e.g., see the $4 D$ Universe visualisation by Dolag et al. 2008). SPLOTCH is a powerful and very flexible ray-tracer software tool which supports the visualisation of large-scale cosmological simulation data (Dolag et al. 2008, 2011; Jin et al. 2010). It is publicly available and continues to be enhanced. A small team is currently working on supporting the visualisation of multi-frequency observational data to achieve realistic $3 \mathrm{D}$ views and fly-throughs of nearby galaxies and galaxy groups.

\section{Acknowledgements}

I like to thank the conference organisers for inviting me to participate in such a fantastic conference in the beautiful city of Vienna. Furthermore, I like to thank my collaborators, in particular Claudio Gheller and Klaus Dolag from the 3D visualisation team, my 
wonderful WALLABY team, and my colleagues Peter Kamphuis and Tiffany Day who continue to improve the $\mathrm{H}$ I models of $\mathrm{M}$ 83. I gratefully acknowledge financial support from the IAU.

\section{References}

Allen, J. T., et al. 2014, MNRAS, in press

Bosma, A. 2004, in Dark Matter in Galaxies, IAU Symposium 220, p. 39

Cappellari, M., et al. 2011, MNRAS, 413, 813

de Blok, W. J. G., et al. 2008, AJ136, 2648

Duffy, A., et al. 2012, MNRAS, 426, 3385

For, B.-Q., Koribalski, B. S., \& Jarrett, T. H. 2012, MNRAS, 425, 1934

Freeman, K., et al. 1977,

Hotan, A., et al. 2014, PASA, in press (arXiv:1409.1325)

Johnston, S., et al. 2008, Experimental Astronomy 22, 151

Jones, K. L., Koribalski, B. S., Elmouttie, M., \& Haynes, R. F. 1999, MNRAS, 302, 649

Józsa, G. I. G. 2007, $A \mathscr{E} A$, 468, 903

Kamphuis, P., et al. 2013,

Kamphuis, P., et al. 2014,

Koribalski, B. S., et al. 2004, AJ, 128, 16

Koribalski, B. S. \& López-Sánchez, Á. R. 2009, MNRAS, 400, 1749

Koribalski, B. S. 2008, in Galaxies in the Local Volume, Astrophysics and Space Science, eds. B. S. Koribalski, and H. Jerjen, p. 41

Koribalski, B. S., et al. 2009, ASKAP proposal

Koribalski, B. S. 2012a, PASA 29, 213

Koribalski, B. S. 2012b, PASA 29, 359

Kreckel, K., et al. 2011, AJ, 141, 204

Obreschkow, D. et al. 2011, ...

Oh, Se-Heon, et al. 2010, ...

Rogstad, et al. 1974, ...

Sánchez, et al. 2012, A\&A, 538, 8

Serra, P., Westmeier, T., et al. 2014, MNRAS, submitted

Serra, P., Koribalski, B. S., Kilborn, V. A., et al. 2015, MNRAS, submitted

Spekkens, K. \& Sellwood, J. A. 2007, ApJ, 664, 204

Krajnovic, et al. 2006,

Verheijen, M., et al. 2008, AIP Conf. Proc. Vol. 1035, p. 265

Warren, B. E., Jerjen, H., \& Koribalski, B. S. 2004, AJ, 128, 1152

Westmeier, T., Braun, R., \& Koribalski, B. S. 2011, MNRAS, 410, 2217

Westmeier, T., Koribalski, B. S., \& Braun, R. 2013, MNRAS, 434, 3511

Westmeier, T., Jurek, R., Obreschkow, D., Koribalski, B. S., \& Staveley-Smith, L. 2014, MNRAS, 438,1176

Wilson, W. E., et al. 2011, MNRAS, 416, 832 